\title{
Análisis de la relación entre creatividad, atención y rendimiento escolar en niños y niñas de más de 9 años en Colombia
}

\section{Analysis of the relationship between creativity, attention and school performance in children and girls over 9 years in Colombia}

\author{
Nubia Cárdenas Ávila \\ Fundación Gustavo Aponte Rojas, Bogotá, Colombia \\ Verónica López-Fernández \\ Universidad Internacional de La Rioja (UNIR), Logroño, España \\ Cristian Camilo Arias-Castro iD \\ Universidad Cooperativa de Colombia, Medellín, Colombia
}

\begin{abstract}
Resumen
Objetivo: El presente artículo de resultados de investigación se llevó a cabo en el marco de la neuropsicología aplicada a la educación. Este artículo busca analizar la relación entre la creatividad, atención visual y auditiva y el rendimiento escolar.

Método: Es un estudio de tipo cuantitativo, no experimental y correlacional; la muestra estuvo conformada por 85 niños y niñas escolarizados, con edad igual o mayor a 9 años y que se encontraran cursando entre cuarto y sexto grado académico. La atención fue evaluada con las subpruebas del dominio de atención, de la batería ENI, correspondientes a atención auditiva y visual, la creatividad a través del test CREA, y el rendimiento académico fue suministrado por la institución educativa por medio del boletín de calificaciones

Resultados: No puntuaron una relación estadísticamente significativa entre la atención y el rendimiento académico, asimismo con la creatividad, difiriendo los hallazgos de León (2008), quien propone los procesos atencionales como predictores del rendimiento académico y de igual manera, los de Corbalán, Martínez, Donolo, Alonso, Tejerina y Limiñana (2003), quienes manifiestan que la inteligencia creativa influye en el procesamiento de información y aprendizaje y, como consecuencia, en el rendimiento; sin embargo, estos resultados pueden obedecer a diferentes variables asociadas al desempeño escolar
\end{abstract}

Conclusiones: El presente estudio puede ser una vía importante para el reconocimiento del valor de la atención en los procesos creativos Además, sugiere la realización de estudios más amplios y centrados en la relación entre la creatividad y el rendimiento para esclarecer más el tipo de relación que tienen.

Artículo de Investigación Copyright 2018 by Psicogente

Correspondencia de autores: nubiacardenas1@hotmail.com veróni-ca.lopez@unir.net critian.ariasc@campusucc.edu.co

Recibido: $26-10-16$

Aceptado: $28-06-17$

Publicado: 01-01-18

Palabras Claves:

Neuropsicología, Creatividad, Atención, Rendimiento Escolar.

\section{Abstract}

Objective: This research paper was conducted based on neuropsychology criteria applied to education. This research aims to analyze the relationship between creativity, attention (visual and auditory attention) and school performance.

Method: It is a quantitative, experimental and cor- relational study. For data collection, 85 schoolchildren, aged 9 years or more, fourth and sixth grade students were sampled. In order to assess auditory and visual attention, subtests related to attention domain, through ENI battery was used; also, creativity was assessed through CREA test, and $t$

Results: statistically, there were no a significant relationship between attention and academic performance, as well as creativity, differing in this way from Leon findings (2008), who proposes attention processes as academic performance predictors and also, from those ones such as: Corbalán, Martinez, Donolo, Alonso, Tejerina and Limiñana (2003) who consider that information and learning process are influenced by creative intelligence; however, these results may obey different variables associated with school performance

Conclusion: This study may be an important tool in order to recognize the value of the attention and creative processes. In addition, it suggests broader studies focus on the relationship between creativity and academic performance.

Key words: Neuropsychology, Creativity, Attention, School performance

Como citar este artículo (APA):

Hernández, L. (2018). Perfil sociodemográfico y académico en estudiantes universitarios respecto a su autoeficacia académica percibida. Psicogente, 21(39), 35-49. http://doi.org/10.17081/psico.21.39.2820 


\section{INTRODUCCIÓN}

¿Existe una relación entre la creatividad, la atención y el rendimiento escolar en niños y niñas de más de 9 años incluidos en el sistema escolar?, o mejor, ¿qué pasa con los estudiantes en el aula, en su atención, creatividad y rendimiento escolar?, ¿son la atención y la creatividad variables facilitadoras del rendimiento escolar? Siendo la creatividad un recurso humano de mucha importancia en lo que respecta a la evolución de nuestro futuro como especie y como ser cultural, no se le ha dado el lugar dentro del currículo escolar (Corbalán \& Limiñana, 2010). En este sentido, el estudio de Jáuregui (2013) hace evidente la necesidad de impulsar la creatividad afirmando que el $100 \%$ de los docentes cree que la actividad docente puede desarrollar la creatividad, el $62 \%$ considera que la creatividad es de vital importancia para los alumnos y el $52 \%$, que la creatividad es muy importante en el desarrollo del trabajo en el aula.

De Zubiría (2014) analiza la situación educativa en Colombia y al respecto concluye que la fórmula para mejorarla pasaría por fomentar la creatividad y las habilidades sociales. Plantea menos extensión en los contenidos que se enseñen y más profundización para que los estudiantes logren pensar (pensamiento convergente: búsqueda, selección, gestión, procesamiento de datos, y pensamiento divergente: aplicabilidad, transformación, creatividad), crear (proceso creativo) y solucionar, siendo estas tres habilidades parte del proceso creativo que dan respuesta y vía de solución a la disonancia existente entre la metodología de enseñanza del siglo pasado y la del nuevo milenio, así como a la confusión o poca claridad de situaciones en el aula de desatención, indisciplina, desmotivación y hasta trastorno por Inatención e Hiperactividad. Un aprendizaje donde se subestime la creatividad y el rol activo de estudiante, el proceso atencional va afectar el rendimiento escolar.

El sistema educativo está llamado a romper paradigmas de enseñanza para potenciar, descubrir capacidades y talentos, por lo que el desarrollo de las capacidades no puede dejarse a la casualidad y espontaneidad. Por ello este estudio aborda la evaluación de la atención, la creatividad y el rendimiento escolar y su correlación en aras a que este conocimiento pueda emplearse para mejorar la práctica educativa

Definir la creatividad ha sido producto de la evolución, la noción y complejización del término, que se da según la escuela y autor, entre ellos el psicoanalítico, cognitivo, psicométrico, social y ecológico. Todos los planteamientos han sido necesarios e importantes en el momento histórico que emerge, para lograr una precisión, comprensión integral y compleja del término, que siguiendo a Huidobro (2004), que realiza un recorrido evolutivo y cronológico de los conceptos de creatividad, es un recorrido excelso.

Una de las definiciones más aceptadas viene de la mano de Runco y Jaeger (2012) que afirman que la creatividad requiere tanto de originalidad como de eficacia y validación. De esta forma, la creatividad se define con mucha frecuencia en términos de productos, aunque también se ha estudiado desde diferentes enfoques como el ambiente, las características de las personas y el proceso creativo (Lim \& Plucker, 2001; Torres y Granados, 2014). 
La creatividad es un proceso que comprende aspectos cognitivos, de producciones creativas mediadas y útiles en un contexto determinado por lo que es útil retomar a Rodríguez (2011), que la define como la "capacidad que poseen las personas para producir ideas originales, a partir de estas, originan materiales nuevos, tomando siempre como referencia el contexto social en el que ocurren" (p.46). El concepto que se complementa y profundiza con los planteamientos de Huidobro (2004), añadiendo la creatividad como implicación de tres rasgos: el intelectual, el personal y el motivacional, los cuales cobran pertinencia desde la neuropsicología de la creatividad ya que son diversas las áreas cerebrales que están implicadas en el proceso creativo, así como la interconexión del lóbulo frontal con otras áreas del córtex, diferentes lóbulos y áreas subcorticales como el sistema límbico, los ganglios basales, núcleos de la base y el cerebelo (Jordan, 2004). La activación de estructuras dependerá de las vías aferentes que pueden ser visual, auditiva, táctil, propioceptiva (sensibilidad-interpretación de estímulos y contextos) de la forma del procesamiento (cognición del proceso creativo) y vía eferente (oral, tonal, escrita, gráfica, motora) de las cuales emerge el producto creativo.

En el ámbito educativo que nos compete, es relevante reseñar la importancia especialmente del contexto en la creatividad. En este sentido, partiendo de los estudios pioneros de Amabile (1986, 1988), Ryan y Deci (2000), se sitúan Nickerson, Stenberg y Lubart (como se citan en Navarro, 2008, p.49). Así Nickerson (1999) sugiere que los elementos que pueden favorecer la creatividad pasan por aspectos como: afirmar propósitos e intenciones. En otras palabras, es necesario un trabajo continuo para que la creatividad se haga posible, construir habilidades básicas en tres niveles: el lenguaje, sistemas estructurados para la solución de problemas y ejecución de proyectos independientes autodirigidos, adquirir conocimientos específicos de los dominios, estimular la curiosidad, etc.

Sobre cómo se relacionan la creatividad y el rendimiento académico, diversos autores destacan la importancia de la creatividad en la consecución de un alto rendimiento académico (Limiñana, Bordoy, Juste \& Corbalan, 2010; Gralewski \& Karwowski, 2012). Sin embargo, en un estudio con alumnos de conservatorio, AlonsoLacasta, Martínez-Monteagudo y Martín-Lobo (2015) hallaron una relación negativa y estadísticamente significativa entre la creatividad y el rendimiento académico. Estos hallazgos son muestra de la controversia actual que existe aún en este tópico de estudio en la actualidad.

La atención al igual que la creatividad posee un desarrollo evolutivo a nivel conceptual, el cual parte de un modelo lineal para transformarse en un complejo sistema que actúa en red neuronal por lo que son denominados estos modelos multidimensionales y afirman que la atención es un estado neurocognitivo cerebral de preparación que precede a la percepción y la acción, y es el resultado de una red de conexiones corticales y subcorticales de predominio hemisférico derecho. La atención focaliza selectivamente la consciencia para filtrar el constante fluir de la información sensorial, resolver la competencia entre los estímulos para su procesamiento en paralelo, y reclutar y activar las zonas cerebrales para temporizar las respuestas apropiadas.

La atención está integrada por los componentes, proceso sensorial proveniente del exterior e interior del organismo cuando está en vigilia, la cual se percibe y bombardea al individuo de 
información, siendo este incapaz de capturarla en su totalidad, por lo que debe filtrar o seleccionar y es ahí donde cobra relevancia la atención a nivel neuronal, neuroquímico y de estructuras cerebrales y de manera paralela la participación simultánea de procesos motores y motivacionales. Por lo que estaríamos hablando de diferentes tipos de acciones, a saber: 1) Sistema de Activación Retícula Ascendente (SARA)-Tallo cerebral (Vigilia); 2) Hemisferios cerebrales, sincronizados por lóbulos prefrontales; 3 ) Sistema límbico y sus estructuras (tono emocional y afectivo) (GarcíaOgueta, 2001).

El proceso inicia con: 1) El sistema Abajo-Arriba que implica una recepción de estímulos por vías aferentes (audición, vista, tacto y propioceptivo) y del medio externo, a través de los sentidos y sus receptores los estímulos se convierten en impulsos nerviosos que llegan al tronco del encéfalo, cerebelo, tálamo y sustancia activadora reticular ascendente (SARA) en donde un grupo de neuronas mielinizadas procesan eficazmente para dar una respuesta contextualizada y oportuna; este proceso se da a nivel subcortical y se desarrolla entre los 0 y 6 años de edad; 2) El sistema ArribaAbajo es el sistema que le precede, el cual se da a nivel de las cortezas y se constituye en un nivel de respuesta o eferente teniendo un protagonismo la integración entre cortezas visual, auditiva y lóbulo frontal a nivel de funciones ejecutivas (reflexión, planeación, ejecución) e interpretaciones emocionales y afectivas para lograr asignar un recurso atencional a la tarea.

Este proceso tiene una integración hemisférica, siendo el hemisferio izquierdo el encargado de recepcionar los estímulos del mundo exterior y el hemisferio derecho conjuga el tono emocional, afectivo a nivel interno para dar un contenido y hacer una marca de aprendizaje, en memoria a largo plazo con un contenido emocional positivo o negativo según la experiencia: sin atención la percepción, memoria y aprendizaje no tiene lugar o empobrece (Blázquez-Alisente, Paúl-Lapedriza \& Muñoz-Céspedes, 2004; Colmenero, Catena \& Fuentes, 2001).

La atención es vital para el aprendizaje y desarrollo ya que permite atender a los estímulos importantes, por ejemplo, en el aprendizaje de la lectura y escritura atención visual (identificación de grafemas y su posición) y auditiva (conciencia fonológica, discriminación de fonemas) son de especial prioridad y según la tarea a realizar participa en grados diferente las áreas corticales y subcorticales a nivel cerebral.

Los mecanismos atencionales son específicos y complejos. Así, los mecanismos que interfieren para que se fije la atención a los rostros (Kagan, 1970; Spiendler, Carlotto, Ogliari y Giordani, 2015). son diferentes a los que se activan en situaciones de predilección de estímulos sonoros (Berg \& Berg, 1979). De esta forma, la información que ingresa por el canal auditivo se ordena a nivel temporal y su duración es corta, en contraste con la información visual que es más rica en cuanto a organización visoespacial y su duración es más larga; esta información ingresa por el sistema visual en especial la retina y llega a áreas visuales occipitales y temporales, especialmente la corteza temporal inferior (Hernández, 2008).

Entendiendo la atención como un proceso psicológico básico y relacionado con las variables de este estudio, algunos autores destacan la relación entre las alteraciones atencionales, el aprendizaje y el rendimiento académico (González-Maya, 2015; Thapar, Cooper, Jefferies \& Stergiakouli, 2012), 


\section{Luis Fernando Hernández Jácquez}

asimismo, la conexión entre la atención, sus estrategias y el rendimiento académico (TejedorTejedor, González-Gonzáles \& García-Señorán, 2008).

En un estudio de la relación entre la atención y la ansiedad, manifestaron que la ansiedad puede ser un factor determinante en la pérdida de control sobre el foco atencional y, a su vez, esto podría tener un impacto en el rendimiento académico obtenido en los exámenes.

Finalmente, el rendimiento escolar se comprende como un aspecto cuantitativo y cualitativo, es el producto del proceso de enseñanza-aprendizaje, asimismo Chadwick (1979) le considera la expresión de las capacidades del estudiante desarrolladas y actualizadas a través del proceso de enseñanza-aprendizaje, esta se reducir a un número en la mayoría de los casos, y este reflejaría el nivel alcanzado por el estudiante, esta afirmación es pertinente para este ejercicio investigativo en la medida que desde el aula los factores exógenos como el contexto familiar, económico, cultural no se pueden interferir; no obstante, factores endógenos son susceptibles de afectar como lo son los biológicos y en especial neuropsicológicos como la atención y la creatividad y exógenos como las pedagogías pensadas desde una metodología creativa.

\section{MÉTODO}

\subsection{Diseño}

Esta investigación presentada es de tipo cuantitativo, no experimental, transversal, descriptivo y correlacional, teniendo en cuenta que, desde su diseño, no pretende alterar las variables. En cambio, centra sus análisis en la comprensión de los fenómenos tal y como se presentan en el contexto, permitiendo entender sus posibles causas (Bernardo \& Caldero, 2000).

\subsection{Participantes}

La población del estudio se centra en escolares matriculados en el programa de educación no formal de Atención en Contra Jornada Escolar (ACE) de las dos jornadas de la Fundación Gustavo Aponte Rojas.

En concreto, participaron 85 estudiantes que se hallaban cursando $4^{\circ}$ y $5^{\circ}$ de primaria, siendo la media de edad de 10 años, con una desviación típica $(1,259)$, las edades de los evaluados estuvieron divididas entre los 9 y 15 años de edad.

Los criterios de inclusión fueron que los alumnos estuviesen matriculados en el centro y que cumplimentasen un consentimiento informado para participar en el estudio. 


\subsection{Instrumentos}

2.3.1 El Test CREA de Inteligencia Creativa de Corbalán, Martínez, Donolo, Alonso, Tejerina y Limiñana (2003).

Permite apreciar la inteligencia creativa a través de una evaluación cognitiva de la creatividad individual a partir de la generación de preguntas escritas sobre un estímulo (imagen), se usa en niños, adolescentes y adultos, cuenta con baremación para las tres edades y se ha trabajado en población española y argentina. Su aplicación es grupal o individual y tiene un máximo de duración de cuatro minutos desarrollándola y 10 con instrucciones. La actividad consistió en realizar el mayor número de preguntas respecto a una imagen que se entregó durante cuatro minutos.

Cabe anotar que autores como Limiñana et al. (2010) utilizaron este test para estudiar la relación entre la creatividad, habilidades intelectuales y estilos de respuesta y sus implicaciones en el rendimiento académico. Según los autores de la prueba, esta se ajusta a los estándares y tiene una fiabilidad alta para evaluación de la creatividad. En relación a la validez, los estudios confirman al test CREA como una herramienta de medición de la creatividad con validez concurrente y discriminante.

\subsubsection{Batería Neuropsicológica ENI de Matute, Rosselli, Ardila y Ostrosky (2007).}

Del dominio atención y el subdominio atención visual (cancelación de dibujos y letras) y el subdominio atención auditiva (serie de números en progresión y regresión), esta prueba ha sido trabajada con población latina (México y Colombia). El procedimiento para atención visual fue entregar una hoja donde aparecen conejos grandes y pequeños, el estudiante al darle la indicación de iniciar deberá marcar la mayor cantidad de conejos grandes. Respecto a otra de las tareas, la cancelación de letras se siguió el mismo procedimiento, pero debía marcar una " $X$ " cada vez que encontrara una " $A$ " seguida de una " $X$ ", que se ejecutó durante un minuto cada subtest. En relación al subdominio atención auditiva se da al participante una serie de números y el niño debe repetirlos, en un segundo momento se da los números y debía repetirlos en regresión, es decir escucha 7 y 2 y la respuesta es 2 y 7 , aproximadamente 2 minutos.

Rendimiento escolar: Recopilación de información a partir del boletín de calificaciones de los niños y niñas de las asignaturas básicas (Español, Matemáticas, Sociales y Ciencias Naturales), conversión y homogeneización de los datos para procesarlos.

\subsection{Procedimiento}

Respecto a la aplicación de los instrumentos, previo consentimiento informado, cabe decir que se realizó de manera grupal (12 a 18 niños y niñas), con un tiempo de duración aproximado de 45 minutos por grupo. Se lograron aplicar las pruebas empleando dos días de aplicación.

Específicamente, en primer lugar, se aplicó la prueba de creatividad, posteriormente la prueba de atención y por último, se recogió la información correspondiente al rendimiento en las materias comentadas anteriormente. Respecto a la prueba CREA y ENI, se siguieron las instrucciones de los 
instrumentos propuestas por los autores. Las pruebas se aplicaron en una sala sin distracciones ni ruidos y la aplicación se desarrolló con normalidad.

\subsection{Análisis de datos}

Una vez aplicados los instrumentos, se procedió a la elaboración de base de datos en Excel y análisis estadístico por medio del programa SPSS versión 22 (se efectuó primero el análisis descriptivo y posteriormente el correlacional, empelando el estadístico de Pearson).

\section{RESULTADOS}

Se presentan los resultados desde los objetivos planteados, por lo cual la capacidad creativa, el nivel atencional y rendimiento escolar de los niños y niñas es el primer objetivo y de orden descriptivo. En la Tabla 1 se puede ver la media de cada variable, la cual corresponde al número de aciertos, datos que guardan relación con la moda; en cuanto a la desviación típica los datos no guardan relación, indicando dispersión en concordancia a la media.

Tabla 1

Estadísticos Descriptivos de las Variables

\begin{tabular}{lcccrc}
\hline \multicolumn{1}{c}{ VARIABLES } & MEDIA & MODA & D.T & MIN & MÁX \\
\hline CREATIVIDAD & 7,41 & 6 & 3,32 &, 00 & 15,00 \\
ATENCIÓN VISUAL & 32,50 & 34 & 8,19 & 17,50 & 53,00 \\
ATENCION AUDITIVA & 7,00 & 7 & 1,34 & 1,50 & 7,50 \\
RENDIMIENTO ESCOLAR & 2,20 & 3 &, 53 & 1,00 & 3,00 \\
\hline
\end{tabular}

Nota: D.T.: Desviación Típica; Mín.: Mínimo; Máx.: Máximo

En la Tabla 2 se observan los datos por variables y edad, siendo de especial interés el bajo nivel en atención visual en modalidad dibujos para todas las edades.

Tabla 2

Media por edad de subvariables de Atención

\begin{tabular}{|c|c|c|c|c|c|c|c|c|c|c|c|c|c|c|c|c|c|c|}
\hline \multirow{4}{*}{ ATENCIÓN } & \multicolumn{18}{|c|}{ EDAD } \\
\hline & \multicolumn{3}{|c|}{$N=21$} & \multicolumn{3}{|c|}{$N=35$} & \multicolumn{3}{|c|}{$\mathrm{N}=17$} & \multicolumn{3}{|c|}{$N=7$} & \multicolumn{3}{|c|}{$N=3$} & \multicolumn{3}{|c|}{$\mathrm{N}=2$} \\
\hline & \multicolumn{3}{|c|}{9} & \multicolumn{3}{|c|}{10} & \multicolumn{3}{|c|}{11} & \multicolumn{3}{|c|}{12} & \multicolumn{3}{|c|}{13} & \multicolumn{3}{|c|}{15} \\
\hline & $M$ & $P$ & $\mathrm{~N}$ & $M$ & $P$ & $N$ & $M$ & $P$ & $\mathrm{~N}$ & $M$ & $P$ & $\mathrm{~N}$ & M & $P$ & $\mathrm{~N}$ & M & $P$ & $\mathrm{~N}$ \\
\hline VISUAL DIBUJO & 22 & 26 & B & 28 & 37 & M & 30 & 37 & $M$ & 29 & 16 & B & 34 & 37 & $M$ & 22 & 0 & B \\
\hline VISUAL LETRAS & 34 & 84 & $A$ & 38 & 75 & $A$ & 44 & 84 & $A$ & 42 & 63 & $M$ & 41 & 50 & $M$ & 45 & 50 & $M$ \\
\hline AUDITIVA D.P. & 6,2 & 75 & A & 7.4 & 95 & $A$ & 8 & 98 & $A$ & 7.8 & 95 & $A$ & 7.6 & 91 & A & 8 & 95 & $A$ \\
\hline AUDITIVA D.R. & 4.7 & 84 & $A$ & 6.1 & 99 & $A$ & 5.6 & 95 & $A$ & 6.7 & 98 & $A$ & 7 & 99 & $A$ & 7 & 98 & $A$ \\
\hline
\end{tabular}

Respecto a los objetivos correlacionales se analizaron mediante el estadístico de correlación de Pearson y arrojaron que no hay relación significativa entre las variables rendimiento escolar/creatividad y rendimiento escolar y atención, negándose la hipótesis de correlación positiva y estadísticamente significativa entre la atención y rendimiento escolar, y la creatividad y el rendimiento escolar; las Figuras 1 y 2 presentan de manera detallada los resultados. En el caso concreto de la Figura 1 se evidencia el gráfico de dispersión entre la atención visual y el rendimiento escolar, la cual no muestra correlación entre las variables. 


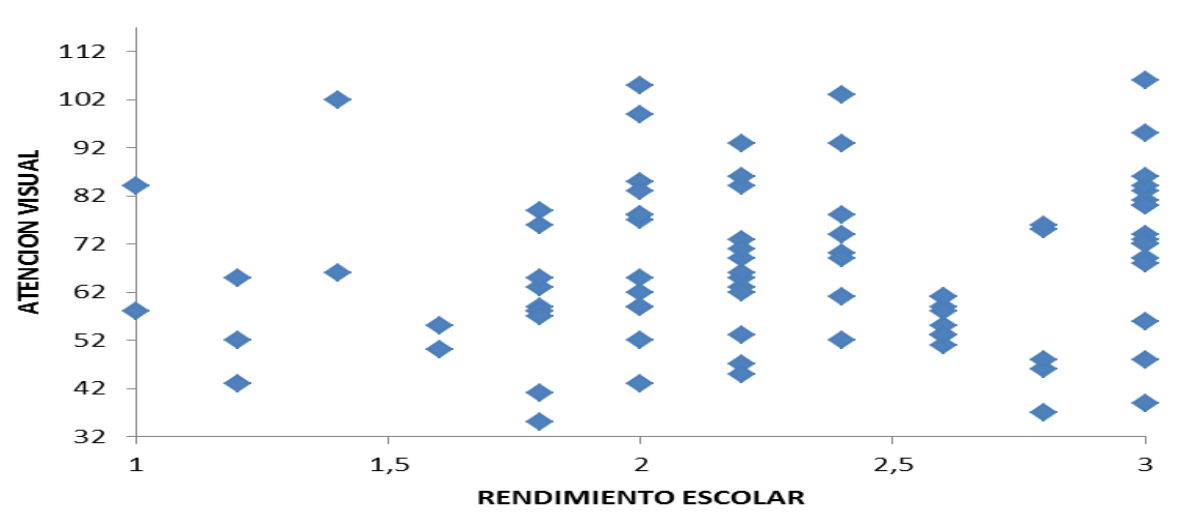

Figura 1. Atención visual y rendimiento escolar

La hipótesis plantea si existe correlación estadísticamente significativa entre Atención (Visualauditiva) y Rendimiento escolar, siendo los datos estadísticos: Correlación de Pearson de 0,110, Probabilidad o p-valor de 0,316, en la comparación con p> a 0,05, la Correlación NO es estadísticamente significativa (datos similares en el análisis individual de atención visual y auditiva).

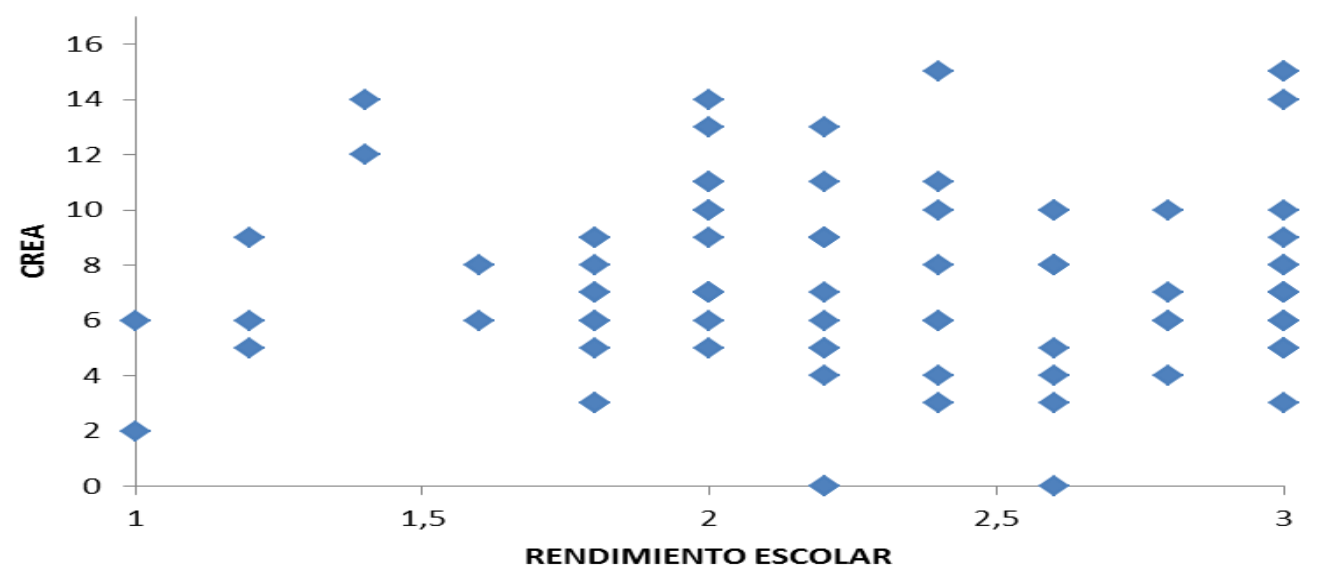

Figura 2. Creatividad y rendimiento escolar

Como se aprecia en la Figura 2 se evidencia el gráfico de dispersión entre la creatividad y el rendimiento escolar, fruto de la recolección de los datos de la muestra. La hipótesis plantea si existe correlación estadísticamente significativa entre creatividad y rendimiento escolar, siendo los datos estadísticos: Correlación de Pearson de 0,026, Probabilidad o p-valor de 0,810, en la comparación con $p>$ a 0,05, la Correlación NO es estadísticamente significativa.

Existe relación estadísticamente significativa entre Creatividad y Atención Visual y Auditiva, las Figuras 3 y 4 presentan detalles al respecto. En ambas figuras se muestra el diagrama de dispersión que refleja un patrón de asociación en la nube de puntos. 


\section{Luis Fernando Hernández Jácquez}

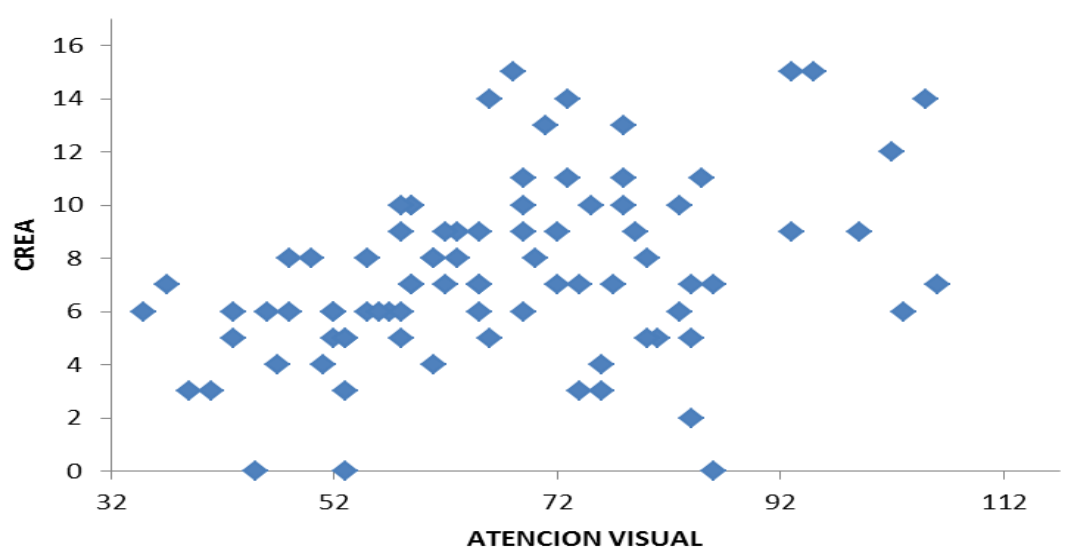

Figura 3. Creatividad y atención visual

Se obtuvo una correlación de Pearson de 0,387 y una probabilidad o p-valor de 0,000, que al compararlo con $p<a 0,05$, indica que Sí hay una correlación entre las dos variables.

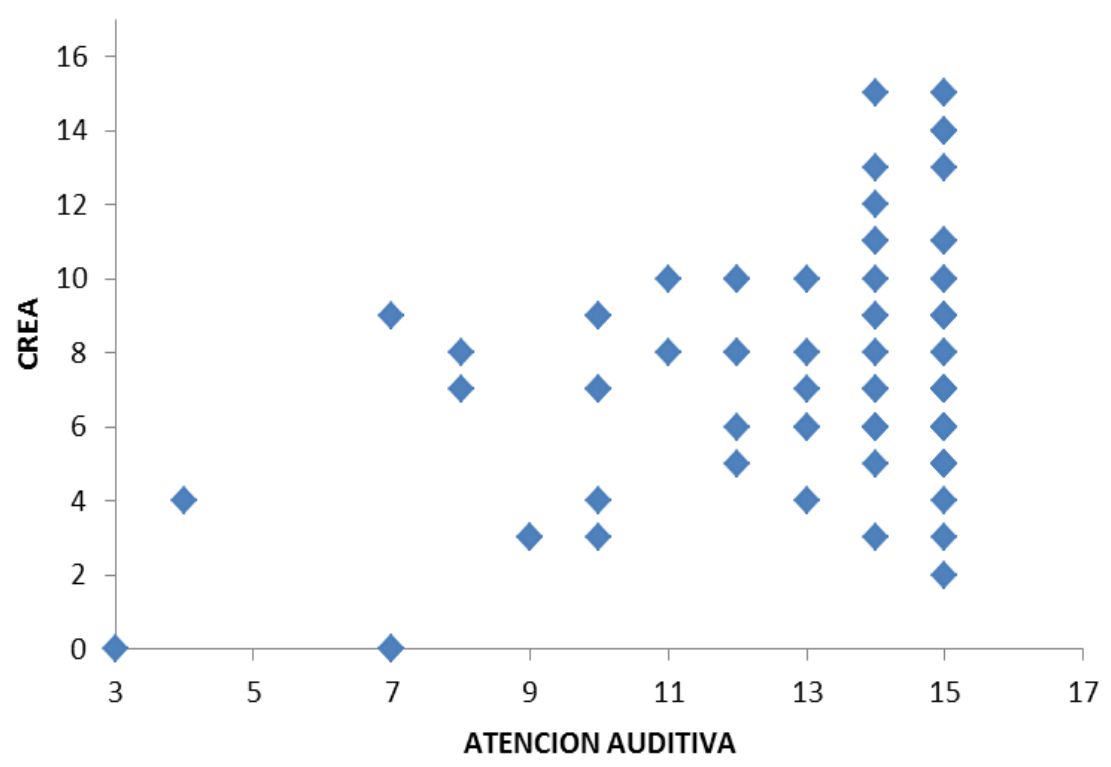

Figura 4. Creatividad y atención auditiva

Se obtuvo una correlación de Pearson de 0,372 y una probabilidad o p-valor de 0,000, que al compararlo con $\mathrm{p}<\mathrm{a} 0,05$, reafirma que Sí hay una correlación entre las dos variables.

\section{DISCUSIÓN}

Los resultados reflejan que los niños y niñas presentaron un desempeño alto en relación a su edad, en la variable creatividad. A pesar de estos hallazgos, cabe anotar que De Zubiría (2014) presentó un análisis de la situación educativa en Colombia, en el cual expresa como solución para la mejora educativa del país, fomentar la creatividad en el aula y los aportes advierten la necesidad y virtud de desplegar en el espacio educativo una pedagogía de la "pregunta" en vez de la "respuesta". De esta forma, los profesores contestan a preguntas que los alumnos no han hecho y es preciso que el 


\section{Luis Fernando Hernández Jácquez}

educador testimonie en los educandos el gusto por la pregunta y el respeto a la pregunta, ya que el silencio es una forma de castrar la curiosidad, sin la cual, no hay creatividad, esto como una realidad educativa, una necesidad y virtud a desarrollar en el aula por parte de sus actores y fomento de la investigación y creatividad desde una teoría ecológica, asimismo, un proceso de enseñanza basado en preguntas puede llegar a generar mayor compromiso por parte del estudiante, independiente de la afinidad o interés por el área de estudio (Bayram, Oskay, Erdem, Özgür, \& Ùenol, 2013).

Al respecto Csikszentmihalyi (1998) expresa que la creatividad no se produce dentro de la cabeza de las personas, sino en la interacción entre los pensamientos de una persona y un contexto sociocultural. Es un fenómeno sistemático, más que individual, por ello resulta interesante preguntarse también: “¿Cuáles son las condiciones que permiten que un aporte personal se constituya en un avance cultural?", como apunta Zapata-Ros (2002) y “¿Cuáles son los sistemas y procesos neuropsicológicos que están interconectados para conseguir estimular la habilidad creadora?", en lenguaje de Jordan (2004).

Respecto a la variable atención auditiva y visual se dio un desempeño bueno, lo que indica a nivel de desarrollo neuropsicológico que hay un adecuado proceso de integración sensorial en vía aferente auditiva y visual en lo que respecta a habilidades de focalización de estímulos sonoros importantes, discriminación auditiva, discriminación de estímulos visuales en forma, posición y secuencia de imagen y memoria a corto plazo, entre otras habilidades (Lázaro \& Berruezo, 2009).

Sin embargo, en atención visual de dibujo el desempeño se dio bajo para el grupo de 9 años, y medio para el grupo de 10 y 11 años, esta tarea implicaba reconocer, focalizar la atención en el tamaño; al respecto el estudio de Gaitán y Rey-Anacona (2013) reveló que los niños y niñas con bajo desempeño en cancelación de dibujos y retención de dígitos en progresión y regresión presentaban diagnóstico de trastorno del cálculo. Lo dicho no ha de servir para hacer suposiciones vagas, pero sí para prestar a futuro especial atención a nuevos ejercicios de investigación en esta línea.

Los resultados progresivos de atención visual según la edad se aprueban con los estudios de RosselliCock et al. (2004) para población colombiana, ya que arrojan que las funciones ejecutivas mejora con la edad, es decir el proceso atencional tiene un desarrollo paulatino

La muestra presenta un desarrollo atencional funcional para el aprendizaje preferentemente de tipo atencional auditivo más que visual. El bajo rendimiento en atención visual de cancelación de dibujos no difiere con los resultados de Berg y Berg (1979), quienes afirman que la información sonora puede ser más corta en su duración y pobre en estímulos, y la visual más rica en estímulos y por ello más duradera, la diferencia entre los resultados radicaría en: 1. La prueba letras tiene mayores herramientas por parte del estímulo para dirigirse a la tarea como son: diferentes posiciones y formas, adicionalmente se está mayormente entrenado en rastreo de letras por el proceso lector que se realiza de manera constante, 2 . La prueba de cancelación de dibujos tiene una única variable estímulo (tamaño), que a la vista es difícil de percibir por lo que invita a un mayor foco, esfuerzo y sostenibilidad atencional.

El rendimiento escolar que presenta la muestra es Básico-Medio, indicando el producto del proceso de enseñanza y aprendizaje mostrando la necesidad de profundizar aspectos que lo afectan desde 
una perspectiva integral y compleja, como señala la Secretaría de Educación Distrital de Bogotá (2010) desde los factores endógenos y exógenos del rendimiento escolar. Al respecto conviene decir que en este estudio, al no disponer de implementación de una herramienta estandarizada que lograra obtener información cualitativa y factorial de esta variable fue una limitante para profundizar.

Respecto a los objetivos correlacionales se encontró que no hay relación significativa entre la atención: visual-auditiva y rendimiento escolar, resultado que va en contravía con León (2008): si se presentan dificultades en el tratamiento de información se influenciará y es un predictor del rendimiento académico. En este punto cabe considerar que los resultados podrían estar afectados por la propia tarea realizada como por ejemplo: 1. Tiempo atencional corto, un minuto para cada tarea, 2 . El grado de motivación, interés y competitividad no medidas que emergió para resolver la tarea atencional, ya que la aplicabilidad se dio en grupo, pudo influir en los resultados; 3. Complejidad de la tarea, habilidades cognitivas y redes atencionales implicadas, estas son variables diferentes a las que se dan en una actividad escolar donde la red atencional de vigilancia, que se extiende a 45 minutos o más con didácticas diferentes y diversidad de variables que inciden en el rendimiento escolar.

No hay relación significativa entre creatividad y rendimiento escolar, estos resultados van en contra de los estudios previos de Garaigordobil y Torres (1996) y Mourgues, Tan, Hein, Elliott y Grigorenko (2016), quienes confirman la incidencia de la creatividad en el rendimiento escolar. En esta línea, también Corbalán et al. (2003) encuentra que el déficit en la inteligencia creativa influye en el procesamiento de información y aprendizaje.

El hecho de no encontrar relación entre las variables creatividad y rendimiento podría radicar en que el rendimiento se basa fundamentalmente en actividades convergentes, más que divergentes (Navarro, 2008).

Así que los resultados estadísticos no significativos puede que obedezcan a la técnica y ausencia de herramienta para medir rendimiento escolar, el peso de la creatividad en el rendimiento escolar es nulo, debido a la diversidad de factores intervinientes (personales, cognitivos y motivacionales) y el tipo de creatividad que se mide. Pérez y Campos (2007) hallaron en un estudio una relación entre las dos variables ya que se contemplaron habilidades especiales en los estudiantes de esta carrera en cuanto a creatividad.

Existe relación entre la variable creatividad y atención, los resultados insinúan dos niveles de análisis, el primero corresponde a la creatividad afectada porque si no se poseen las habilidades cognitivas implicadas para plantear preguntas sobre un estímulo visual, el nivel de atención frente a la tarea se ve afectado. Adicionalmente el desarrollo de la atención es esencial para procesos cognitivos, así mismo es un indicador para predecir el nivel cognitivo en la niñez, entre mejor es la capacidad atencional, mejor es la ejecución de las tareas (Hernández, 2008).

El segundo corresponde a estudios que encontraron que, a mayor creatividad, mayor oscilación atencional, amplitud atencional y distractivilidad (Martínez Zaragoza, 2010; Enríquez, Fajardo y Garzón, 2015), lo que indica que la focalización en un estímulo inhibe el pensamiento divergente y 


\section{Luis Fernando Hernández Jácquez}

limita la producción de ideas, en este mismo sentido, Oshin, Gilbert, Illingworth y Bowden (2009) plantean que hay mejor ajuste del foco atencional según la demanda y claridad de la tarea, pues su ambigüedad desenfoca la atención generando lentitud y eficacia.

En definitiva, y para concluir, a pesar de que este estudio tiene sus limitaciones, es una vía importante para reconocer el valor de la atención en procesos tan relevantes y que implican grandes habilidades cognitivas como los procesos creativos. Crear programas basados en la adquisición de la información, puede tener efectos positivos en la creatividad. Futuros estudios han de centrarse en la relación entre la creatividad y el rendimiento para esclarecer más el tipo de relación que tienen y poder emplear la creatividad como optimizador del rendimiento en nuestras clases y procesos de aprendizaje.

Nota de Autores

Derivado del estudio de investigación: Relación entre creatividad, atención y rendimiento escolar en niños-as escolarizados de más de 9 años

\section{REFERENCIAS}

Alonso-Lacasta, R., Martínez-Monteagudo, M. \& Martín-Lobo, P. (2015). Creatividad, atención y rendimiento académico en alumnado de conservatorio profesional. Revista INFAD de Psicología, 1(1), 473-482. DOI: http://dx.doi.org/10.17060/ijodaep.2015.n1.v1.32

Amabile, T. M. (1986). Creativity in context: Update to the social psychology of creativity. US: Westview press.

Amabile, T. M. (1988). A model of creativity and innovation in organizations. Research in organizational behavior, 10(1), 123-167. Disponible en http://www.ijtbm.com/images/short_pdf/Feb_2011_Research_Paper_07.pdf

Bayram, Z., Oskay, Ö. Ö., Erdem, E., Özgür, S. D. \& Úenol, Ú. (2013). Effect of Inquiry Based Learning Method on Students' Motivation. 4th International Conference on New Horizons in Education. Procedia - Social and Behavioral Sciences, 106(2013), 988-996. https://doi.org/10.1016/j.sbspro.2013.12.112

Berg, W.K. \& Berg, K.M. (1979). Psychophysiological development in infancy: state, sensory function, and attention. In J.D. Osofsky (Ed.), Handbook of Infant Development (pp. 283-43). New York: Wiley.

Bernardo, J. \& Caldero, J.F. (2000). Investigación cuantitativa (4); métodos no experimentales. En J. Bernardo y J.F. Caldero, Aprendo a investigar en educación. Madrid: RIALP. S.A.

Blázquez-Alisente, J.L., Paúl-Lapedriza, N. \& Muñoz Céspedes, J.M. (2004). Atención y funcionamiento ejecutivo en la rehabilitación neuropsicológica de los procesos visuoespaciales. Revista de Neurología, 38(5), 487-495. Disponible en https://www.neurologia.com/articulo/2003538

Chadwick, C. (1979). Los adolescentes ante el estudio, causas y consecuencias del rendimiento académico. España: Ed. Fundamentos. 


\section{Luis Fernando Hernández Jácquez}

Colmenero, J., Catena, A. \& Fuentes, L. (2001). Atención visual: Una revisión sobre las redes atencionales del cerebro. Anales de Psicología, 17(1), 45-67. Disponible en https://psiquiatria.com/neuropsiquiatria/atencion-visualuna-revision-sobre-las-redes-atencionales-del-cerebro/

Corbalán, B.J. \& Limiñana, G. (2010). El genio en una botella. El test CREA, las preguntas y la creatividad. Introducción al monográfico, "El test CREA, inteligencia creativa". Anales de Psicología, 26(2), 197-205. Disponible en http://psycnet.apa.org/record/2010-13853-001

Corbalán, F.J., Martínez, F., Donolo, D. S., Alonso, C., Tejerina, M. \& Limiñana, R.M. (2003). Crea. Inteligencia creativa. Una medida cognitiva de la creatividad. Manual. Madrid: TEA Editores.

Csikszentmihalyi, M. (1998). Creatividad: El fluir y la psicología del descubrimiento y la invención. Barcelona: Paidós.

De Zubiría, S. (2014). ¿Cómo mejorar la educación en Colombia? Recuperado de http://www.las2orillas.co/comomejorar-la-educacion-en-86

Enríquez, M., Fajardo, M., Garzón, F. (2015). Una revisión general a los hábitos y técnicas de estudio en el ámbito universitario. Psicogente, 18(33), 166-187. http://doi.org/10.17081/psico.18.33.64

Gaitán, A. \& Rey-Anacona, C. (2013). Diferencias en funciones ejecutivas en escolares normales, con trastorno por déficit de atención e hiperactividad, trastorno del cálculo y condición comórbida. Avances en Psicología Latinoamericana, 31(1), 71-85. Disponible en http://revistas.urosario.edu.co/index.php/apl/article/view/2347

Garaigordobil, M. \& Torres, E. (1996). Evaluación de la creatividad en sus correlatos con inteligencia y rendimiento académico. Universitas Tarraconensis, 18(1), 87-101. Disponible en http://www.sc.ehu.es/ptwgalam/art_completo/tarraco1.PDF

García-Ogueta, M.I. (2001). Mecanismos atencionales y síndromes neuropsicológicos. Revista de Neurología, 32(5), 463-467. Disponible en https://w3.ual.es/ Ifuentes/sindromes.pdf

González-Maya, M.V. (2015). La alteración en las Funciones Ejecutivas en el TDAH y su relación con el rendimiento académico. (Tesis de maestría). Universidad Internacional de La Rioja, España.

Gralewski, J. \& Karwowski, M. (2012). Creativity and school grades: a case from Poland. Thinking skills and Creativity. ERIC, 7(3), 198-208. https://doi.org/10.1016/j.tsc.2012.03.002

Hernández, P. (2008). Desarrollo de los procesos atencionales (Tesis doctoral). Universidad Complutense de Madrid, España.

Huidobro, S. (2004). Una definición de la creatividad a través del estudio de 24 autores seleccionados (Tesis doctoral). Universidad Complutense de Madrid, España. Disponible en http://eprints.ucm.es/4571/

Jáuregui, C.E. (2013). Desarrollo de una guía de Intervención del profesor, para estimular la creatividad en la metodología de resolución de problemas tecnológicos (Tesis de maestría). Universidad de Rioja, España. Disponible en https://reunir.unir.net/handle/123456789/1480

Jordan, V. (2004). Funciones cognitivas del lóbulo frontal. Revista de Neuropsicología, 39(2), 178-182. Disponible en https://www.neurologia.com/articulo/2004254

Kagan, J. (1970). Attention and psychological change in the young child. Science, 170(3960), 826-832. Disponible en https://www.ncbi.nlm.nih.gov/pubmed/5473415 


\section{Luis Fernando Hernández Jácquez}

Lázaro, A. \& Berruezo, P. (2009). La pirámide del desarrollo humano. Revista Iberoamericana de Psicomotricidad y Técnicas Corporales, 34, 74-103. Disponible en http://www.colegiogloriafuertes.es/articulos/articulo2piramide.pdf

León, B. (2008). Atención plena y rendimiento académico en estudiantes de enseñanza secundaria. European Journal of Education and Psychology, 1(3), 17- 26. http://dx.doi.org/10.1989/ejep.v1i3.11

Lim, W. \& Plucker, J. (2001). Creativity through a lens of social responsibility: Implicit theories of creativity with Korean samples. Journal of Creative Behavior, 35(2), 115-130. http://dx.doi.org/10.1002/j.2162-6057.2001. tb01225.x

Limiñana, R., Bordoy, M., Juste, G. \& Corbalan, J. (2010). Creativity, intelectual abilities and response styles: implacation for academic performance in the secondary school. Anales de Psicología, 26(2), 212-219. Disponible en http://revistas.um.es/analesps/article/view/109081

Martínez-Zaragoza, F. (2010). Creatividad: impulsividad, atención y arousal. Del rasgo al proceso. (Tesis doctoral). Universidad de Murcia, España. Disponible en http://www.tesisenred.net/handle/10803/11027;jsessionid=199E6170DB0769FD3F27F4A2B3DFDB57

Matute, E., Rosselli, M., Ardila, A. \& Ostrosky, F. (2007). ENI: evaluación neuropsicológica infantil. Guadalajara-México: Manual Moderno-UNAM- Universidad de Guadalajara,

Mourgues, C., Tan, M., Hein, S., Elliott, J.G. \& Grigorenko, E.L. (2016). Using creativity to predict future academic performance: An application of Aurora's five subtests for creativity. Learning and Individual Differences, 51, 378-386. https://doi.org/10.1016/j.lindif.2016.02.001

Navarro, J. (2008). Mejora de la creatividad en el aula de Primaria (Tesis Doctoral). Universidad de Murcia, España.

Nickerson, R. S. (1999). Enhancing Creativity. In R.J. Sternberg, Handbook of creativity (p. 392). New York: Cambridge University Press.

Oshin, O., Gilbert, A., Illingworth, J. \& Bowden, R. (2009, September). Action recognition using randomised ferns. In Computer Vision Workshops (ICCV Workshops), 2009 IEEE 12th International Conference on (pp. 530-537). IEEE.

Pérez, F. \& Campos, A. (2007). Creatividad y rendimiento académico en estudiantes de bellas artes. Revista GalegoPortuguesa de Psicoloxía e Educación, 15(2), 127-133. Disponible en https://minerva.usc.es/xmlui/handle/10347/459

Rodríguez, M.F. (2011). Contribuciones de la neurociencia al rendimiento de la creatividad humana. Revista: Arte, $\begin{array}{lllll}\text { Individuo } \quad \text { y } & \text { Sociedad, } & \text { 45-54. } & \text { Disponible en }\end{array}$ https://revistas.ucm.es/index.php/ARIS/article/viewFile/36253/35103

Rosselli-Cock, M., Matute-Villaseñor, E., Ardila-Ardila, A., Botero-Gómez, V. E., Tangarife-Salazar, G. A., EcheverríaPulido, S. E., Arbeláez-Giraldo, C., Mejía-Quintero, M., Méndez, M., Villa-Hurtado, P. \& Ocampo-Agudelo, P. (2004). Evaluación Neuropsicológica Infantil (ENI): una batería para la evaluación de niños entre 5 y 16 años de edad. Estudio normativo colombiano. Revista de Neurología, 38(8), 720-731. Disponible en https://www.neurologia.com/articulo/2003400

Runco, M. A. \& Jaeger, G. J. (2012). The standard definition of creativity. Creativity Research Journal, 24(1), 92-96. http://dx.doi.org/10.1080/1 0400419.2012.650092 


\section{Luis Fernando Hernández Jácquez}

Ryan, R. M. \& Deci, E. L. (2000). Self-determination theory and the facilitation of intrinsic motivation, social development, and well-being. American psychologist, 55(1), 68-78. http://dx.doi.org/10.1037/0003066X.55.1.68

Secretaría de Educación Distrital de Bogotá (2010). Factores que influyen en el rendimiento escolar. Recuperado de http://evaluacion.educacionbogota.edu.co/files/Factores\%20que\%20influyen\%20en\%20el\%20rendimiento $\% 20$ escolar.pdf

Spiendler, S., Carlotto, M., Ogliari, D., Giordani, K. (2015). Estressores ocupacionais em psicólogos clínicos brasileiros. Psicogente, 18(33), 104-116. http://doi.org/10.17081/psico.18.33.59

Tejedor-Tejedor, F.J., González-Gonzáles, S.G. \& García Señorán, M. (2008). Estrategias atencionales y rendimiento académico en estudiantes de secundaria. Revista Latinoamericana de Psicología, 40(1), $123-132$. http://dx.doi.org/10.14349/rlp.v40i1.351

Thapar, A., Cooper, M., Jefferies, R. \& Stergiakouli, E. (2012). What causes attention deficit hyperactivity disorder? Archives of disease in childhood, 97(3), 260-265. http://dx.doi.org/10.1136/archdischild-2011-300482

Torres, P. \& Granados, D. E. (2014). Procesos cognoscitivos implicados en la comprensión lectora en tercer grado de educación primaria. Psicogente, 17(32), 452-459.

Zapata-Ros, M. (2002). ¿Puede el aprendizaje no ser creativo? Y otras reflexiones a propósito de CREATIVIDAD. EI fluir y la psicología del descubrimiento y la invención, de Mihaly Csikszentmihalyi, y de la glosa que sobre este libro hace Cristina Rinaudo. Revista de educación a distancia, 4, 1-4. Disponible en http://revistas.um.es/red/article/view/25261

Está obra está bajo: Creative commons attribution 4.0 international license. El beneficiario de la licencia tiene el derecho de copiar, distribuir, exhibir y representar la obra y hacer obras derivadas siempre y cuando reconozca y cite la obra de la forma especificada por el autor o el licenciante

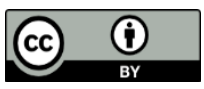

